\title{
BMJ Open A pilot randomised controlled trial of metacognitive therapy for prolonged grief
}

\author{
Jenine Anne Wenn, Moira O'Connor, Robert T Kane, Clare Samantha Rees, \\ Lauren J Breen
}

To cite: Wenn JA, O'Connor M, Kane RT, et al. A pilot randomised controlled trial of metacognitive therapy for prolonged grief. BMJ Open 2019;9:e021409. doi:10.1136/ bmjopen-2017-021409

- Prepublication history for this paper is available online. To view these files, please visit the journal online (http://dx.doi. org/10.1136/bmjopen-2017021409).

Received 6 January 2018 Revised 3 October 2018 Accepted 4 October 2018
Check for updates

(C) Author(s) (or their employer(s)) 2019. Re-use permitted under CC BY-NC. No commercial re-use. See rights and permissions. Published by BMJ.

School of Psychology, Faculty of Health Sciences, Curtin University, Perth, Western Australia, Australia

Correspondence to Dr Jenine Anne Wenn; jenine.wenn@postgrad.curtin. edu.au

\section{ABSTRACT}

Objectives Prolonged grief disorder is associated with significant distress and impairment and thus efforts to improve treatments are essential. The present pilot study tested the efficacy and feasibility of group Metacognitive Grief Therapy (MCGT) designed specifically for prolonged grief symptomatology to reduce the psychological distress and impaired function resulting from bereavement. Design/participants Twenty-two bereaved adult participants with prolonged grief symptomatology were randomised to a wait-list control $(n=10)$ or an intervention condition $(n=12)$ with a 3-month and 6-month follow-up. The wait-list control group was offered treatment after the post-test assessment. Intervention Participants attended six group MCGT sessions that ran for 2 hours per week.

Outcome measures A primary outcome measure of prolonged grief symptomatology and secondary outcome measures of depression, anxiety, rumination, metacognitive beliefs and quality of life were taken pretreatment and post-treatment for both groups and at the 3-month and 6-month follow-up for the intervention group. A Generalised Linear Mixed Model was used to assess treatment efficacy.

Results Post-treatment intent-to-treat analyses showed MCGT reduced prolonged grief symptomatology (Cohen's $d=1.7)$, depression $(d=1.3)$, anxiety $(d=0.8)$, stress $(d=1.0)$, rumination $(d=0.9)$ and increased quality of life $(d=0.6)$, and these effects were maintained at the 3-month and 6-month follow-ups. No prepost between-group differences were found in metacognitive beliefs. However, a large significant effect was identified at the 3-month and 6-month follow-ups $(\mathrm{d}=1.0)$.

Conclusion The results show promise for the utility of group MCGT for reducing psychological distress and promoting quality of life. Additionally, the results underscore the need for a full randomised controlled trial of group MCGT, which may be an important addition to the treatment armamentarium available to support people with prolonged grief.

Trial registration number ACTRN12613001270707; Results.

Original protocol BMJ Open 2015;5:e007221. doi:10.1136/bmjopen-2014-007221

\section{INTRODUCTION}

Prolonged grief disorder (PGD) is a debilitating condition experienced by

\section{Strengths and limitations of this study}

Metacognitive Grief Therapy was found to be a short and effective treatment for prolonged grief with low attrition.

- The effect sizes achieved by the six session Metacognitive Grief Therapy programme were large and it was well received by bereaved people, providing support for the utility of the programme for people experiencing prolonged grief symptomatology.

- The small sample size and no comparative treatment underscore the need for a larger randomised controlled trial of Metacognitive Grief Therapy.

approximately $7 \%$ of the bereaved population. ${ }^{2}$ Those experiencing PGD find it difficult to come to terms with the loss, lose their sense of purpose in life, avoid reminders of the loss, become preoccupied with thoughts of the deceased and experience an intense yearning for the deceased that does not remit with time. ${ }^{34}$ This intense distress is associated with social and occupational impairment and high levels of self-neglect (eg, self-care and nutrition), health issues and substance use. ${ }^{5}$

Bereaved people have often been diagnosed with major depression or post-traumatic stress disorder following a traumatic death. ${ }^{6}$ However, factor analytic studies have established PGD symptomatology is distinct from anxiety and depression and independently associated with considerable morbidity and increased suicidality. ${ }^{4}{ }^{5}$ Encouragingly, the recent inclusion of persistent complex bereavement disorder in the fifth edition of the Diagnostic and Statistical Manual of Mental Disorders (DSM-5) ${ }^{7}$ and the inclusion of PGD in the next International Classification of Diseases (ICD) ${ }^{8}$ means more people experiencing prolonged symptoms with grief will be identified. However, current effective evidence-based therapies for PGD are limited. ${ }^{4}$ PGD is known to persist without adequate treatment, ${ }^{10}$ thus treatments 
specifically for PGD to ameliorate the loss of functioning and psychosocial distress resulting from bereavement are needed, and research is required to test the efficacy of these interventions.

Emerging evidence supports the use of psychosocial interventions designed to target PGD directly using components of cognitive behavioural therapy. ${ }^{4}$ For example, a pioneer study by Shear $e t a l^{11}$ trialled a 20-session treatment comprising exposure therapy, interpersonal therapy and motivational interviewing specifically designed for complicated grief, and a 12-session intervention comprising six sessions of cognitive restructuring and six sessions of exposure, ${ }^{12}$ both demonstrated significant reductions in PGD symptomatology. Furthermore, comparative studies trialling exposure therapy against cognitive restructuring and behavioural activation ${ }^{12} 13$ found exposure therapy produces greater reductions in PGD than behavioural activation or cognitive restructuring. However, several individual sessions of exposure therapy are required ${ }^{11}{ }^{14-16}$ during treatment due to the uniqueness of each person's death story and, as such, it may not be feasible for services that can only offer support groups or a limited number of sessions. Thus far, group interventions for PGD have not produced the effect sizes achieved by individual interventions and have experienced substantial attrition. ${ }^{14-18}$

The above studies support the use of therapeutic interventions for PGD, but raise questions about what more could be done to tailor interventions to target the mechanisms underlying PGD directly. Unlike other psychological disorders, such as depression and anxiety, PGD involves mental anguish over many realistic thoughts about the events surrounding the death and the consequences of the loss (eg, shock the deceased will not be returning, financial stressors), rather than maladaptive thoughts about perceived threat. ${ }^{19}$ Research has identified excessive worry about the uncertainty of the future and repetitive thinking about the events and emotions related to the loss both intensify PGD symptomatology. ${ }^{20-23}$ Recent research has demonstrated that the use of rumination as a coping process may be associated with poor bereavement outcomes ${ }^{24}$ with a growing body of literature suggesting that rumination may be used as an anxious avoidant coping process by the bereaved as a means to avoid the reality of the loss. ${ }^{25} 26$ Therefore, a treatment for PGD that addresses the maladaptive coping processes used to manage loss-related thoughts may be more effective than addressing the content of maladaptive thoughts.

Within the large family of cognitive behavioural therapies are contemporary approaches that do not emphasise modification of the content of thoughts. One such approach is metacognitive therapy (MCT), underpinned by the metacognitive model of psychological disorder, which is framed within a general self-regulatory executive function model. ${ }^{27}$ The central premise of the metacognitive model is that psychological disorders arise not from negative or dysfunctional thoughts per se but rather from the way individuals respond to such thoughts. Psychological disorders are associated with the cognitive attentional syndrome (CAS), ${ }^{27}$ a particular style of thinking and responding to thoughts and/or feelings.

The CAS involves chains of worry/rumination, threat monitoring and maladaptive coping behaviours (eg, thought suppression and avoidance behaviours) and is driven by positive and negative metacognitive beliefs. For example, a traumatic memory may be responded to with rumination, driven by the metacognitive belief that rumination is necessary to find answers to what happened. This way of responding maintains threat monitoring and prevents adaptive emotional processing. ${ }^{28}$ MCT aims to interrupt the CAS to enable adaptive emotional processing to occur and to modify unhelpful metacognitive beliefs. MCT focuses on modifying unhelpful thought processes such as rumination and worry. Various therapeutic techniques are used in MCT, such as detached mindfulness, attention training technique (ATT) and behavioural experiments. ${ }^{29}$

As MCT focuses on unhelpful thought processes that maintain psychological disorders, we felt that it may offer great promise in the treatment of PGD. To investigate this further, we recently conducted a qualitative study of the metacognitive beliefs occurring in the context of PGD. ${ }^{30} \mathrm{~A}$ number of metacognitive beliefs were maintaining rumination, worry and avoidance coping behaviours. For instance, bereaved people were found to have positive metacognitive beliefs that rumination/ worry (repetitive negative thinking) was a useful means to 'make sense' of the death, cope or find answers; negative metacognitive beliefs that grief-related thoughts were uncontrollable or harmful and positive metacognitive beliefs about avoidance (eg, the need to push specific thoughts away). Grief-specific metacognitive beliefs were also identified, such as the importance of using rumination to preserve memories of the deceased; emotional regulation as a means to manage grief and show respect/love; avoidance behaviours to evade the reality of the loss and the use of symbolic objects/rituals to maintain a connection with the deceased. Metacognitive beliefs may keep bereaved people focused on loss issues, which prevent them from integrating the loss into their lives and planning for the future. The findings from the study exemplified the need for the development of PGD treatments targeting the metacognitions that drive unhelpful coping processes.

The Dual Process Model of Coping with Bereavement proposes that pathological grief occurs when exclusive focus is on either the loss or restoration (eg, secondary stressors resulting from the death such as financial stressors, employment and child care).$^{19}$ Therefore, treatment for PGD may be more effective if the focus is shifted away from the content of thoughts and toward the modification of unhelpful metacognitive beliefs about coping processes that impede the integration of the loss into their lives. Thus, the aim of this pilot study was to explore the efficacy and feasibility of a group Metacognitive Grief 


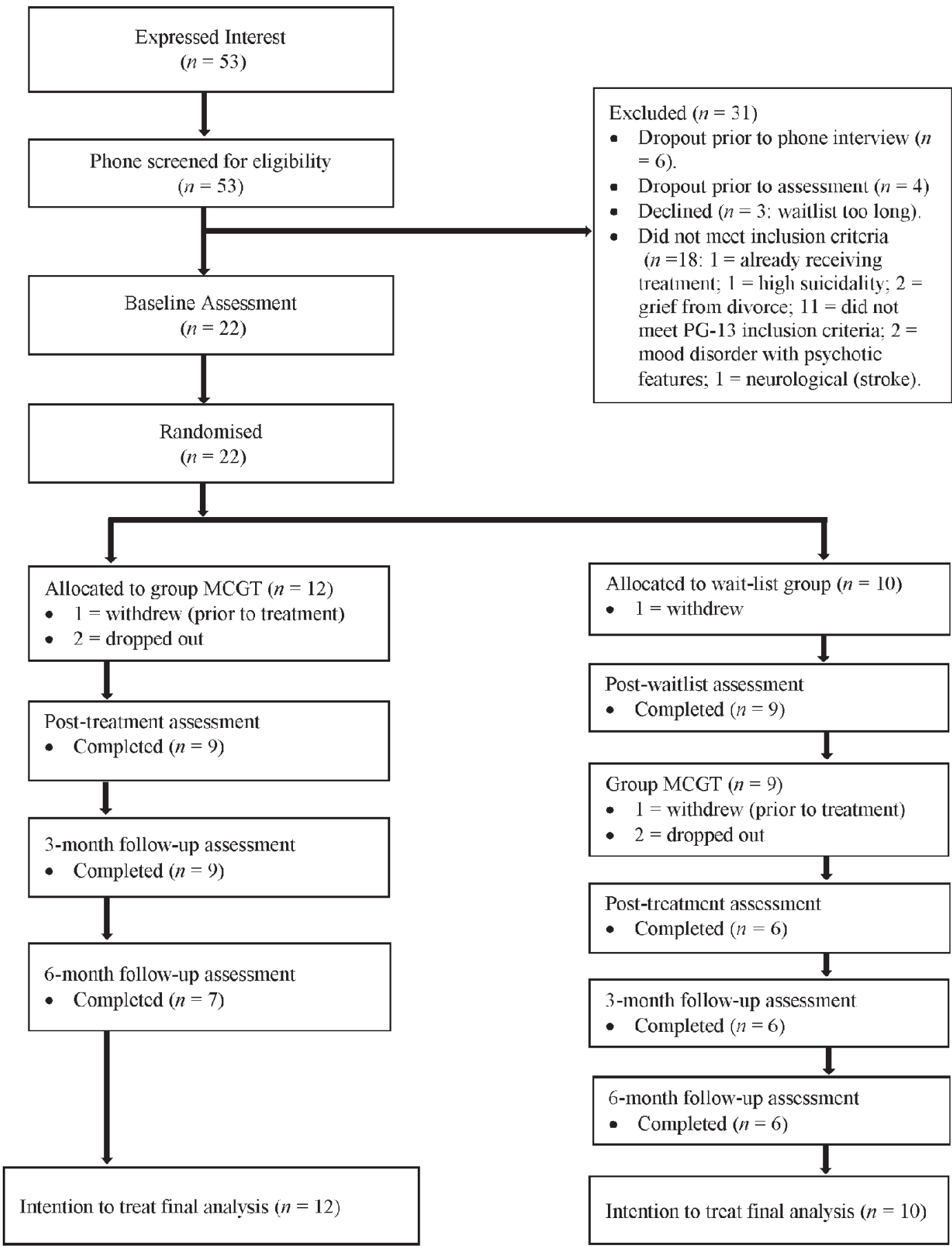

Figure 1 Consolidated Standards of Reporting Trials flow chart of participants through the study. MCGT, Metacognitive Grief Therapy; PG-13, Prolonged Grief Disorder Scale.

Therapy (MCGT) for PGD via a preliminary randomised controlled trial.

\section{HYPOTHESES}

1. The intervention group will report significantly greater prepost decreases in PGD symptomatology, unhelpful metacognitions, rumination, depression, anxiety and stress, and a significantly greater increase in quality of life, compared with the wait-list (WL) group.
2. For the intervention group, postintervention levels in PGD symptomatology, unhelpful metacognitions, rumination, depression, anxiety, stress and quality of life will be maintained at the 3-month and 6-month follow-up.

\section{METHOD}

\section{Trial design}

The trial comprised a randomised treatment MCGT/ WL control group design with a 3-month and 6-month 
follow-up ${ }^{31}$ (figure 1). The WL control group was offered MCGT after the post-test assessment, for ethical reasons, to limit participant distress. The sample size obtained in this study was small ( $\mathrm{n}=22$ intent-to-treat $/ \mathrm{n}=18$ completers). However, small samples including a WL control group are considered sufficient for a pilot trial. ${ }^{32}$

\section{Patient and public involvement}

Bereavement guidelines and empirical research with bereaved carers clearly point to the necessity of interventions for PGD. ${ }^{433}$ Bereaved adults experiencing prolonged grief symptomatology were the target participants for this project. Interviews were conducted with key stakeholders (bereavement specialists and bereaved people) to ensure that the intervention was appropriate, applicable and feasible, and to get input from the target population directly. The data gleaned from the interviews informed the development of MCGT and the project design and aims. Although participants in the trial were not involved in the recruitment for the study, they assessed the appropriateness and acceptability of MCGT. All participants received a summary of the project outcomes.

\section{Participants}

Adult bereaved individuals (age range 38-78 years) at least 6 months post the loss of a significant other. Inclusion criteria: prolonged grief symptomatology (PGD Scale cut-off score of $\geq 26$, based on previous prevalence rates $)^{334}$ or functional impairment (4/5 criteria; A, B, $\mathrm{C}$ and $\mathrm{E}$ ), so that people with elevated or diagnosable levels of PGD could participate (10 people met PGD diagnosis with scores ranging from 38 to 52, 11 people met the cut-off score with scores ranging from 27 to 37 and 1 met criteria A, B, C and E with a score of 23); English speaking; written informed consent; participants taking medication (antidepressants/mood stabilisers), need to have commenced 1 month prior to enrolment and the dosage remain the same during the research. Participant exclusion criteria: concurrent psychological intervention, substance abuse, high suicidal ideation or pre-existing psychotic/bipolar/neurological disorder as measured by the Mini International Neuropsychiatric Interview (MINI)..$^{35}$

\section{Intervention content and delivery}

An outline of the MCGT programme is outlined in table 1 and described in the published $\mathrm{PhD}^{36}$ and protocol. ${ }^{31}$ MCGT was adapted from the MCT techniques used by Wells ${ }^{37}$ for emotional disorders, to ensure it aligned with MCT and comprised the elements required to target unhelpful metacognitive processes effectively. MCGT also incorporated grief specific examples to which bereaved people could relate. ${ }^{30}$ It involves an ATT that aims to promote the use of detached mindfulness ${ }^{29}{ }^{37}$ to develop people's metacognitive control to disengage from emotionally laden thoughts and unhelpful behaviours, to build coping strategies and process the loss. Thus, it changes the way distressing thoughts are processed

\begin{tabular}{ll}
\hline Table 1 & Outline of 'metacognitive grief therapy' \\
\hline Session & Content \\
\hline 1 & Psychoeducation on grief/prolonged grief \\
& Formulate metacognitive model of grief \\
& Enhance awareness of metacognitive beliefs \\
& involved in grief \\
& Introduce the importance of self-monitoring \\
& grief-related metacognitive beliefs \\
& Explore the link between metacognitions, \\
& emotions and behaviour \\
& Identify and challenge unhelpful grief-related \\
& metacognitive beliefs \\
Introduce and practice detached mindfulness & Introduce rumination/worry postponement \\
& Detached mindfulness practice \\
Introduce and practice attention training & Challenge positive metacognitive beliefs about \\
& rumination/worry \\
& Practice attention training \\
& Challenge negative metacognitive beliefs \\
about grief-related thoughts/emotions/images \\
being uncontrollable and positive beliefs about \\
rumination \\
Explore metacognitive beliefs underlying the \\
need to avoid the reality of the loss \\
Introduce situational attentional refocusing \\
Challenge unhelpful metacognitive beliefs about \\
avoiding social and leisure activities \\
Practice attention training \\
Programme review \\
Maintenance planning \\
Practice attention training \\
Relapse prevention
\end{tabular}

rather than addressing the content. This bodes well for treating grief in a group setting, as individuals with PGD have been found to be less satisfied with group participation due to co-rumination (conversations with others about the loss). ${ }^{38}$ The intervention comprised six, 2-hour sessions, 1 day per week. Each session was facilitated by the primary researcher and a co-facilitator (both provisionally registered psychologists under supervision) at the university psychology clinic or a community centre. A catch-up booster session was offered to participants who missed a session. No differences were found between treatment and treated control participants in session attendance $(t(20)=0.89, \mathrm{p}=0.38)$. Session attendance for treatment completers ranged from five to six sessions with $73.3 \%$ completing all six.

\section{Measures}

A demographic information sheet was used to obtain information such as postcode, gender, current psychological treatment, medical history, nationality, employment status, relationship with the deceased and the date and cause of death. 


\section{Diagnostic interview}

MINI $^{35}$ was used to identify comorbid disorders and assess suicidal risk and ideation. It involves a short structured interview based on the DSM-5 Axis I or ICD-10 criteria and high test-retest reliability $(0.76-0.93)$ and validity ${ }^{39}$ have been reported.

\section{Self-report measures}

The PGD Scale (PG-13) ${ }^{3}$ was used as the primary measure to assess the diagnostic status and severity of PGD. Diagnosis requires endorsement of five criteria: (A) bereavement via the death of a significant other, (B) separation distress, (C) duration $\geq 6$ months, (D) cognitive, emotional and behavioural symptoms and (E) social/ functional impairment. The 13-item scale requires a yes/ no response for two questions and uses a 5-point Likert scale $(1=$ not at all to $5=$ several times a day/overwhelmingly) to assess symptom severity. A total score is achieved by summing the scores (range 11-55). Inclusion in this study allowed for subclinical levels of PGD (eg, $\geq 26$ cut-off or criteria A, B, C and E) as it is best to have as many participants as feasible when testing the efficacy of a new intervention within a homogenous sample. ${ }^{32}$ The PG-13 has good internal consistency (Cronbach's $\alpha=0.82$ ) and incremental validity. ${ }^{40}$ Internal consistency for PG-13 in the current sample was good $(\alpha=0.80)$.

The Depression Anxiety Stress Scales- 21 (DASS-21) ${ }^{41}$ assesses depression, anxiety and stress and has high internal consistency for depression $(\alpha=0.94)$, anxiety $(\alpha=0.87)$ and stress $(\alpha=0.91)$ scales and validity. ${ }^{41}$ In this sample, it had a high internal consistency for depression $(\alpha=0.88)$, anxiety $(\alpha=0.87)$ and stress $(\alpha=0.88)$.

The Utrecht Grief Rumination Scale (UGRS) ${ }^{42}$ measures grief-specific rumination. It has five subscales: thoughts about consequences and meaning of the loss; thoughts about social support; what-if questions; why questions and thoughts about feelings. A total score can be obtained by summing the subscales (range 15-75). The internal consistency $(\alpha=0.90)$ and validity ${ }^{42}$ of this measure have been shown to be excellent. In this sample, the internal consistency of the total UGRS was high $(\alpha=0.89)$.

The Metacognitions Questionnaire-30 (MCQ-30) ${ }^{43}$ was used to measure metacognitive beliefs. It has five subscales: positive beliefs about worry, negative beliefs about worry concerning uncontrollability and danger, low cognitive confidence, need to control thoughts and cognitive self-consciousness. A total score is achieved by summing the subscales (range 30-120). Internal consistency ranges from 0.72 to 0.93 across the subscales with a total internal consistency of 0.93 and a test-retest reliability of $0.75 .{ }^{43}$ In this sample, the internal consistency ranged from 0.76 to 0.98 with a total internal consistency of 0.95 .

The Quality of Life Enjoyment and Satisfaction Questionnaire-18 (Q-LES-Q-18) ${ }^{44}$ assesses general quality of life across the following domains: physical health, subjective feelings, leisure time activity, social relationships and satisfaction with medication. A total score can be obtained by summing the subscales (range 1-5). It has high internal consistency ranging from 0.82 to 0.94 and test-retest reliability ranging from 0.71 to $0.83 .{ }^{44}$ In the current study, it had a high internal consistency of 0.89 and the subscales ranged from 0.71 to 0.89 .

\section{Therapist measure}

The Clinical Global Impression (CGI) ${ }^{45}$ severity and improvement scales were used to rate each participant's pretreatment to post-treatment progress. A global rating of severity in clinical disorders is determined by scores ranging from 1 to 7 (normal to among the most extremely ill) and an improvement is determined by scores ranging from 1 to 7 (very much improved to very much worse). A rating of 1,2 or 3 (very much improved, much improved or minimally improved) indicates response to treatment. It has high inter-rater reliability $(0.87$ to 0.99$)$. The first author and an experienced psychologist (blind to the study) independently completed the scale to limit experimenter effects. The intraclass correlation (ICC), used to assess the inter-rater reliability using the 'two-way mixed model' in conjunction with the 'consistency procedure', ${ }^{46}$ revealed the raters agreed $98 \%$ of the time (95\% CI 0.97 to 0.99$)$.

\section{Procedure}

The participants were recruited through advertisements on the radio/television and in print media/websites/ flyers in shopping centres, bereavement groups, palliative care services and mental health providers. Participants who expressed an interest in the study were screened by telephone and then attended an individual session to complete the MINI. The baseline diagnoses were determined by the interviewer (first author) prior to scoring the self-reported measures to ensure they were blind to participant diagnoses. Supervision with an experienced clinical psychologist (fourth author) confirmed the accuracy of the diagnoses. Eligible participants were randomised prior to assessment by the first author to an intervention or WL control condition using a simple randomisation procedure (computer-generated random number) to avoid research bias in allocation. Randomisation was created using Excel 2013 with 1:1 allocation.

The primary and secondary self-report measures were completed by the intervention group at baseline, immediately following the intervention and at 3-month and 6-month follow-ups. The WL group completed the measures at baseline, after a 6 -week wait, at treatment completion and at 3-month and 6-month follow-ups. All interviews and assessments were administered by the first author, under the supervision of a clinical psychologist (from 2014 to mid-2015). To maintain confidentiality and preserve anonymity, an ID number was used to code the participants' data. The data were securely stored in a password-protected file on the university server that could only be accessed by the research team. Identifying information (ie, demographic information and consent forms) was kept in a locked filing cabinet in a secured university office. Participants were compensated for their time at the post-test and 6-month assessment points with 
a small monetary gift voucher. Participants wishing to drop out of the study were provided appropriate referral information.

\section{Programme integrity and content compliance}

A clinical psychologist with extensive MCT experience (fourth author) trained and supervised the group facilitators (provisionally registered psychologists) and confirmed that the integrity of the draft protocol for the workshops. To assess the integrity of the detailed sessionby-session manualised MCGT programme delivery and to control for protocol adherence, an implementation efficacy checklist was completed independently by the group facilitator and the co-facilitator at completion of each session. The checklist assessed the overall success of the session, preparation (knowledge of materials, organisation), presentation (clarity, pacing, thoroughness) and rapport using a 10-point Likert scale ( $1=$ very poor to $10=$ excellent). The ICC $^{46}$ indicated that the inter-rater reliability on the implementation efficacy checklist was $79 \%$ (95\% CI, 0.66 to 0.87 ). The fourth author, with over a decade of experience in the delivery of MCT, reviewed the session recordings and confirmed adherence to the treatment protocol.

\section{Social validity/feasibility}

The social validity of the programme was measured using a programme satisfaction questionnaire. Participants rated the overall programme (section A); the practicality of the skills taught (section $\mathrm{B}$ ) and provided qualitative feedback (section C). Sections A and B items were rated on a 5-point Likert scale ranging from 1 (not at all) to 5 (very much) and section $\mathrm{C}$ comprised five open-ended questions for qualitative feedback about the components most and least enjoyed. The appropriateness and acceptability of the programme was determined by the questionnaire. Descriptive statistics were used to summarise the quantitative and qualitative responses.

\section{Data analysis}

The independent variables were group condition (MCGT; $\mathrm{WL}$ ) and time. The dependent variable was treatment response, measured by change in scores for PGD symptomatology, depression, anxiety, stress, metacognitions, rumination and quality of life. A Generalised Linear Mixed Model (GLMM) was used to test H1 and H2. The model included one categorical random effect (participant), one categorical fixed effect (group: intervention, control), one ordinal fixed effect (time: pretest, post-test) and the group xtime interaction. A second GLMM was used to test H3, which included one categorical random effect (participant) and one ordinal fixed effect (time: pretest, post-test, 3-month and 6-month follow-up). In order to optimise the likelihood of convergence, a separate GLMM analysis was run for each of the outcome measures (PG-13, DASS-21, MCQ-30, Q-LES-Q-18, UGRS). To conserve statistical power, the alpha correction was applied within groups of conceptually related outcomes. The GLMM 'robust statistics' option was invoked to accommodate any violations of normality and homogeneity of variance. Violations of sphericity were accommodated by changing the covariance matrix from the default of compound symmetry to autoregressive.

Participants were considered to have dropped out if they missed more than two treatment sessions. To determine whether dropouts differed from completers, demographics and baseline outcome measures were compared and any between-group differences identified were controlled by including them as covariates in the GLMM. Partial eta-squared and Cohen's d were used to assess treatment effect sizes. ${ }^{47}$ A reliable change (RC) score was calculated for each participant. The RC score can be interpreted as the degree to which the participant changes on the outcome variable divided by the SE of difference between the time 1 and time 2 scores. An absolute value of the RC score $>1.96$ reflects an $\mathrm{RC} .{ }^{48}$ For participants who demonstrated an RC and their post-test score fell below the clinical cut-off, this participant experienced clinically significant change. ${ }^{48}$

\section{RESULTS}

\section{Sample characteristics and baseline differences}

Of the 53 individuals who expressed interest, 22 (male $\mathrm{n}=1$ and female $\mathrm{n}=21$ ) were eligible and randomised as outlined in CONSORT flow chart in figure 1. Independent-sample t-tests and Fisher's exact tests revealed no significant baseline differences between groups on demographic and clinical profile characteristics (table 2). Four MCGT groups were conducted: group $1=N 7$, of these one participant dropped out due to another participant's grief and completed MCGT individually; group $2=N 4$, of these one participant dropped out due to another participant's grief; group $3=N 4$, of these one participant dropped out due to a family crisis and another was too distressed to be with others and completed MCGT individually and group $4=N 4$. There was no significant difference between the baseline scores for the treatment and WL group on the outcome measures, except on physical health (Q-LESQ-18 subscale) $t(36)=3.29, \mathrm{p}=0.002$, with the MCGT group exhibiting poorer physical health than the control group. No significant difference was found between the baseline scores for participants taking medication compared with not taking medication (all $t(20)<1.89$, p $>0.31$ ).

\section{Treatment effects on primary outcome}

Large and significant group $\times$ time interactions (table 3) were found for PGD severity. MCGT resulted in significant prepost reductions on the severity of PGD symptomatology. In contrast, the control group was found to significantly increase in PGD severity (PG-13; $t(36)=2.06$, $\mathrm{p}=0.05, \mathrm{~d}=0.48)$. At treatment completion, no MCGT participants met the full diagnostic criteria required for PGD diagnosis, while in the control group this number increased from four to five participants. 
Table 2 Demographic and clinical characteristics of intention-to-treat sample

\begin{tabular}{|c|c|c|c|c|c|}
\hline \multirow[b]{3}{*}{ Characteristic } & Treatment & Control & Total & \multirow[b]{2}{*}{ Significance } & \multirow[b]{3}{*}{$P$ values } \\
\hline & $(n=12)$ & $(n=10)$ & $(n=22)$ & & \\
\hline & Range, mean (SD) & Range, mean (SD) & Mean (SD) & $t(20)$ & \\
\hline Age & $41-78,61(12.3)$ & $38-75,62(10.4)$ & $62(11.2)$ & 0.23 & 0.82 \\
\hline \multirow[t]{2}{*}{ Time (months) post death } & $11-72,29(18.8)$ & $6-60,19(18.5)$ & $24(18.4)$ & 1.37 & 0.19 \\
\hline & n (\%) & n (\%) & $\mathbf{N}(\%)$ & \multicolumn{2}{|l|}{ Fisher's exact $p$} \\
\hline \multicolumn{6}{|l|}{ Relationship to deceased } \\
\hline Partner & $8(67)$ & $10(100)$ & $18(82)$ & \multicolumn{2}{|l|}{0.07} \\
\hline Parent & $3(25)$ & 0 & $3(14)$ & \multicolumn{2}{|l|}{0.14} \\
\hline Child & $1(8)$ & 0 & $1(5)$ & \multicolumn{2}{|l|}{0.55} \\
\hline \multicolumn{6}{|l|}{ Employment status } \\
\hline Employed & $3(25)$ & $2(20)$ & $5(23)$ & \multicolumn{2}{|l|}{0.59} \\
\hline Self-funded retiree & $2(17)$ & $1(10)$ & $3(14)$ & \multicolumn{2}{|l|}{0.57} \\
\hline Pension & $7(58)$ & $7(70)$ & $14(64)$ & \multicolumn{2}{|l|}{0.45} \\
\hline \multicolumn{6}{|l|}{ Living situation } \\
\hline Living alone & $5(42)$ & $7(70)$ & $12(55)$ & \multicolumn{2}{|l|}{0.19} \\
\hline Cohabitating & $7(58)$ & $3(30)$ & $10(46)$ & \multicolumn{2}{|l|}{0.19} \\
\hline \multicolumn{6}{|l|}{ Death type } \\
\hline Sudden & $3(25)$ & $4(40)$ & $7(32)$ & \multicolumn{2}{|l|}{0.38} \\
\hline Chronic illness & $7(58)$ & $6(60)$ & $13(59)$ & \multicolumn{2}{|l|}{0.64} \\
\hline Accident & $2(17)$ & 0 & $2(9)$ & \multicolumn{2}{|l|}{0.29} \\
\hline \multicolumn{6}{|l|}{ Clinical profile } \\
\hline PGD & $6(50)$ & $4(40)$ & $10(46)$ & \multicolumn{2}{|l|}{0.49} \\
\hline \multicolumn{6}{|l|}{ MINI } \\
\hline Depression & $8(67)$ & $3(30)$ & $11(50)$ & \multicolumn{2}{|l|}{0.1} \\
\hline Anxiety disorder & $9(75)$ & $5(50)$ & $14(64)$ & \multicolumn{2}{|l|}{0.22} \\
\hline Post-traumatic stress disorder & $1(8.3)$ & $1(10)$ & $2(9)$ & \multicolumn{2}{|l|}{0.71} \\
\hline
\end{tabular}

Data are expressed as range, means (SD) or as number (rounded \%).

MINI, Mini International Neuropsychiatric Interview; PGD, prolonged grief disorder.

Table 3 Outcome variable means (SD) and within-group effect sizes at baseline and post-treatment/postwait-list for the intervention and control conditions (intention-to-treat analyses)

\begin{tabular}{|c|c|c|c|c|c|c|c|c|c|}
\hline \multirow[b]{2}{*}{ Measure } & \multicolumn{2}{|l|}{$\begin{array}{l}\text { Group } \\
\text { xtime }\end{array}$} & \multirow[b]{2}{*}{ Partial $\eta^{2}$} & \multicolumn{3}{|c|}{ Intervention condition } & \multicolumn{3}{|c|}{ Control condition } \\
\hline & $F(1,36)$ & $P$ values & & Baseline & $\begin{array}{l}\text { Post- } \\
\text { treatment }\end{array}$ & $\begin{array}{l}\text { Cohen's } \\
\text { d }\end{array}$ & Baseline & Postwait-list & $\begin{array}{l}\text { Cohen's } \\
\text { d }\end{array}$ \\
\hline$P G-13$ & 28.19 & $<0.001^{*}$ & 0.44 & $40.00(7.03)$ & $26.36(9.08)$ & 1.68 & $36.80(7.34)$ & $40.40(7.81)$ & -0.48 \\
\hline Depression & 14.75 & $<0.001^{*}$ & 0.29 & $21.17(9.39)$ & $9.86(8.21)$ & 1.28 & $18.80(9.93)$ & $24.43(11.26)$ & -0.53 \\
\hline Anxiety & 10.56 & $0.003^{*}$ & 0.23 & $16.00(10.81)$ & $8.20(9.46)$ & 0.77 & $12.40(10.91)$ & $18.36(13.06)$ & -0.5 \\
\hline Stress & 15.20 & $<0.001^{*}$ & 0.3 & 20.50 (10.46) & $11.19(7.27)$ & 1.03 & $16.80(8.63)$ & $22.13(11.23)$ & -0.53 \\
\hline MCQ-30 & 0.83 & 0.37 & 0.02 & $64.00(18.46)$ & 54.31 (14.69) & 0.58 & $62.70(20.55)$ & $60.57(24.25)$ & 0.09 \\
\hline Q-LES-Q-18 & 6.96 & $0.01^{*}$ & 0.16 & $2.96(0.62)$ & $3.43(1.00)$ & 0.56 & $3.29(0.47)$ & $3.07(0.70)$ & -0.37 \\
\hline UGRS & 6.50 & $0.02^{*}$ & 0.15 & $43.67(10.88)$ & $31.68(15.14)$ & 0.91 & $44.60(14.51)$ & 45.69 (14.55) & -0.08 \\
\hline
\end{tabular}

*Denotes a significant difference.

PG-13, Prolonged Grief Disorder Scale; MCQ-30, Metacognitions Questionnaire; Q-LES-Q-18, Quality of Life Enjoyment and Satisfaction Questionnaire; UGRS, Utrecht Grief Rumination Scale. 


\section{Treatment effects on secondary outcomes}

Large significant group $\times$ time interactions were found for depression, anxiety, stress, rumination (UGRS) and quality of life (Q-LES-Q-18; see table 3). Non-significant group xtime interactions were found for metacognitive beliefs (MCQ-30). The least significant difference (LSD) contrasts showed that group MCGT resulted in significant prepost reductions on measures of depression, anxiety, stress, rumination and quality of life. In contrast, the control group had a significant increase in anxiety $(t(36)=2.67, \mathrm{p}=0.01, \mathrm{~d}=0.50)$, stress $(t(36)=2.43, \mathrm{p}=0.02$, $\mathrm{d}=0.53)$ and a significant decrease in physical health (Q-LES-Q-18 subscale; $t(36)=2.15, \mathrm{p}=0.04, \mathrm{~d}=0.49$ ), but no significant changes in depression, metacognitive beliefs or rumination.

\section{Maintenance of change at 3-month and 6-month follow-up}

As outlined in table 4, non-significant interaction effects indicated that the treated controls made comparable gains to the treatment group after receiving MCGT across pretreatment, post-treatment and follow-up on all outcome variables, except on the UGRS. An LSD contrast revealed the treatment group had large significant prepost reductions that were maintained at the 3-month follow-up with a further significant reduction at the 6-month follow-up, while the treated controls showed a large significant prepost reduction with a further significant reduction at the 3-month follow-up that returned to post-treatment level at the 6-month follow-up. Therefore, the means for the treatment and treated control groups were pooled.

The large significant main effects of time found for PGD severity, depression, anxiety, stress, metacognitive beliefs, rumination and quality of life suggest gains were made from MCGT and maintained at the 3-month and 6-month follow-ups. The length of pretreatment wait-times did not influence treatment outcomes (all $r(15)<0.30, \mathrm{p}>0.28$ ). No significant differences were identified between participants taking medication and those not taking medication on the treatment outcomes (all $t(57)<1.96, \mathrm{p}>0.06)$.

\section{Reliable change and clinically significant change}

Fisher's exact one-sided tests revealed that a significantly greater proportion of MCGT participants $(\mathrm{p}=0.005)$ showed prepost reliable change in PGD severity (PG-13; $67 \%)$ than the control group (22\%). There were no longer significant group differences once the control group received MCGT $(\mathrm{p}=0.71)$, suggesting both groups made similar gains following treatment. At the 3-month follow-up, $73 \%$ of participants experienced a reliable change and $53 \%$ experienced a clinical significant change (PG-13 criterion of $<26$ cut-off) in PGD severity. At the 6-month follow-up, $85 \%$ experienced a reliable change and $62 \%$ experienced a clinically significant change.

\section{Therapist measure of treated participants' progress (CGI)}

The GLMM non-significant group $\times$ time effect $(F(3$, $50)=1.43, \mathrm{p}=0.24)$ indicated treatment and treated

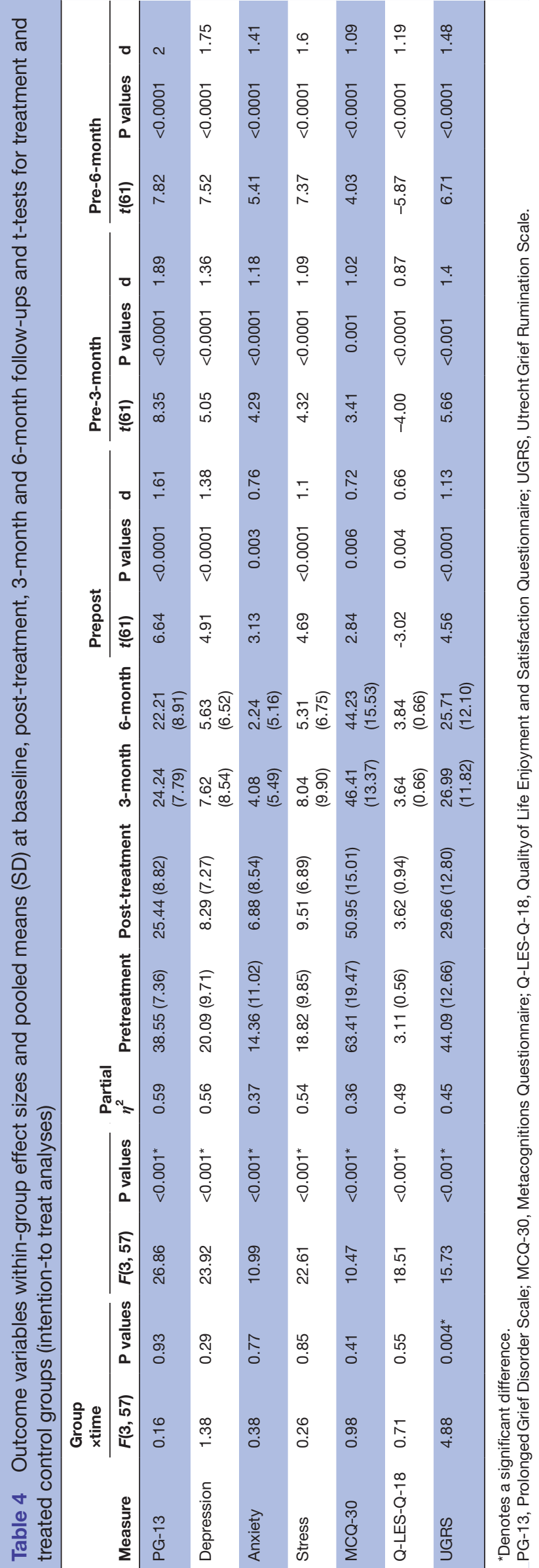


controls did not differ in their illness severity or recovery. There was a significant effect for time $(F(3,54)=155.80$, $\mathrm{p}<0.001)$ for the pooled groups. LSD contrasts revealed significant treatment gains were made from prepost $(t(54)=8.49, \mathrm{p}<0.001, \mathrm{~d}=3.63)$, pre- 3 months $(t(54)=12.48$, $\mathrm{p}<0.001, \mathrm{~d}=4.19)$ and pre- 6 months $(t(54)=17.88, \mathrm{p}<0.001$, $\mathrm{d}=5.07)$. The pretreatment severity of participants' illness ratings ranged from 4-6 (moderately ill to severely ill; mean $=4.77, \mathrm{SD}=0.75,95 \%$ CI 4.39 to 5.14 ) to $3-1$ (minimally to very much improved) at post-test (mean $=2.27$, $\mathrm{SD}=0.62$, $95 \%$ CI 1.96 to 2.58), the 3-month follow-up (mean $=1.77, \mathrm{SD}=0.68,95 \% \mathrm{CI} 1.43$ to 2.11 ) and 6-month follow-up (mean=1.35, $\mathrm{SD}=0.59,95 \%$ CI 1.03 to 1.66 ).

\section{Programme integrity and content compliance}

The co-facilitators rated the delivery of the programme higher than the facilitator (mean $=9.0, \mathrm{SD}=0.94$, range $6-10$; mean $=8.4, \mathrm{SD}=0.69$, range $6-9$ ). Descriptive statistics indicated that the overall ratings for the success of the group was high (mean $=8.2, \mathrm{SD}=0.90)$, as were ratings of session preparation (mean $=8.7, \mathrm{SD}=0.65)$, presentation $($ mean $=8.5, \mathrm{SD}=0.78)$ and rapport $(\mathrm{mean}=9.01, \mathrm{SD}=0.55)$.

\section{Social validity/feasibility}

A feasibility analysis was conducted on the Programme Satisfaction Questionnaire completed by 12 out of the 15 treatment completers. Participant ratings ranged from four to five (agree to very much agree $=100 \%$ ). Descriptive statistics indicated all participants: looked forward to the programme each week (mean $=4.8, \mathrm{SD}=0.45$ ); found the sessions easy to understand (mean $=4.7, \mathrm{SD}=0.49$ ); the programme was useful in everyday life (mean $=4.8$, $\mathrm{SD}=0.39)$; were satisfied with the content covered (mean=4.8, $\mathrm{SD}=0.39)$; the programme was effective in helping them (mean=4.6, $\mathrm{SD}=0.52)$; they noticed positive changes in their lives (mean $=4.5, \mathrm{SD}=0.52$ ); overall, rating of the programme (mean $=4.8, \mathrm{SD}=0.39$ ). Positive qualitative feedback indicated participants found the workshop helpful: "I have skills to help me move forward" ... "Understanding myself and being able to cope, accept and let go of my feelings". Negative feedback suggested some participants would have preferred more sessions.

\section{DISCUSSION}

The results of this pilot study provide preliminary evidence for the efficacy and feasibility of a metacognitive-based group intervention in treating PGD. Participants showed significant improvements in PGD symptomatology, depression, anxiety, stress, rumination and quality of life following MCGT in comparison to participants in the $\mathrm{WL}$ control condition. In contrast, people in the WL exhibited significantly poorer grief symptomatology, anxiety, stress and physical health at post-test, which may have contributed to the significant between-group differences observed. We cannot offer any concrete explanation as to why the WL control group participants deteriorated as we did not directly investigate this.
However, significant improvements in prolonged grief symptomatology, depression, anxiety, stress, repetitive negative thinking, quality of life and metacognitive beliefs were identified in the pooled groups and these improvements were found to be maintained or increased at follow-up. The large effect sizes observed on the PG-13 at post-treatment $(\mathrm{d}=1.68)$ and the uncontrolled 3-month and 6-month follow-up assessments $(\mathrm{d}=1.89 ; \mathrm{d}=2.00)$ provide evidence supporting the efficacy of the six-session MCGT programme. The mean reduction in prolonged grief symptomatology by the 6-month follow-up was $42 \%$ with $85 \%$ of participants identified as having a good level of functioning and minimal symptoms (on the CGI and $\mathrm{RC}$ ). The participants indicated that they found the programme enjoyable, informative and applicable to their everyday lives.

A strength of the study was the longitudinal design which controlled for effects such as time and individual differences, thus providing a clearer picture of the effect of MCGT on outcomes over time. The treatment effects in this study were achieved within a short period of time using a manualised protocol delivered by novice facilitators. It is unlikely the large effects observed can be attributed to extraneous factors or spontaneous recovery due to the stable baselines and various lengthy durations of PGD observed across the sample prior to the intervention.

Unfortunately, the sample size obtained in this study was relatively small and comprised predominantly female participants grieving the loss of a spouse and, as such, may not be representative of all people with PGD. This may be due to the homogeneity of the group, the commonly held assumption that grief is a normal process that does not require intervention ${ }^{49}$ or because we were drawing from a small proportion of a remote population (Perth, Western Australia). We could not compare the intervention and WL groups at the two follow-up periods because it was not deemed ethical to keep bereaved people waiting a long period of time for the intervention.

Furthermore, the results may have been influenced by the primary researcher who administered the assessments and conducted the therapy. As such, participants may have exaggerated treatment gains to please the therapist. ${ }^{50}$ It is also possible the improvements could be due to mere participation in a supportive group rather than the intervention itself, as no comparative treatment was used. However, it is important to note that PGD symptoms have been shown to persist and become chronic over time without targeted intervention (as identified in our WL group), yet this pattern was not demonstrated at the 6-month follow-up. The WL control condition were also found to make comparable gains after receiving MCGT, providing further support for the need for early intervention for people experiencing PGD symptomatology. ${ }^{40}$

Additionally, there was a lag in improvements on the metacognitive belief questionnaire, which may be because it was designed primarily to assess metacognitive beliefs about worry and, as such, may not have been specific 
enough to pick up changes in this sample. The development and inclusion of a grief-specific metacognitive questionnaire in future research with a larger sample size may be useful. Another explanation for the lack of change on the MCQ-30 at post-test could be that the ability to identify and challenge grief-related metacognitive beliefs may take time to develop. Moreover, MCGT was devised and delivered independently of the originators and thus it is possible larger outcomes may have been achieved by a facilitator with extensive experience in the delivery of MCT. Unfortunately, the small sample obtained meant that the study was underpowered for the mediation analyses originally planned..$^{31}$ However, the significant posttest reductions in rumination, lend support for MCGT to target maladaptive metacognitive beliefs effectively.

Despite these limitations, the participants indicated that they were very satisfied with the intervention and felt that the intervention was helpful in reducing their distress. This was confirmed by the results, which indicated that MCGT produced statistically significant improvements on the key outcome measures. These encouraging results support the need for larger randomised studies of the effects of MCGT on PGD compared with another treatment condition and a WL control group with a longer follow-up period. The study also supports the call for treatments to target PGD symptomatology directly and to identify the underlying maladaptive coping mechanisms. This is important given that many PGD interventions have several components and the efficacy of these components remains obscure. ${ }^{4}$ The clinical implications from this study are that PGD treatments incorporating techniques that target maladaptive metacognitions directly may be integral for optimal treatment gains to be achieved for all bereaved individuals.

Acknowledgements The authors would like to acknowledge the bereaved participants who participated in this research.

Contributors JAW, MO'C, LJB, CSR were involved in the study conception, and developed the research design, methodology, intervention and implementation. The research was conducted by JAW under the supervision of MO'C, LJB and CSR. CSR provided clinical expertise and MCT training. RTK provided statistical input into the design, data analysis and interpretation. JAW drafted the manuscript. All authors edited drafts of the manuscript and approved the final manuscript.

Funding JAW was a Doctor of Philosophy (PhD; Clinical Psychology) candidate and used the PhD budget for the manuscript's preparation. LJB is supported by the Australian Research Council (DE120101640).

Competing interests None declared.

Patient consent Not required.

Ethics approval Ethics approval was obtained from Curtin University Human Research Ethics Committee (approval number HR 41/2013).

Provenance and peer review Not commissioned; externally peer reviewed.

Data sharing statement All quantitative data are available via Curtin Universities Research Data Repository (espace@Curtin; D0I: 10.4225/06/5ae682f2ce82d).

Open access This is an open access article distributed in accordance with the Creative Commons Attribution Non Commercial (CC BY-NC 4.0) license, which permits others to distribute, remix, adapt, build upon this work non-commercially, and license their derivative works on different terms, provided the original work is properly cited, appropriate credit is given, any changes made indicated, and the use is non-commercial. See: http:// creativecommons.org/licenses/by-nc/4.0/.

\section{REFERENCES}

1. Aoun SM, Breen LJ, Howting DA, et al. Who needs bereavement support? A population based survey of bereavement risk and support need. PLoS One 2015;10:e0121101.

2. Kersting A, Brähler E, Glaesmer $\mathrm{H}$, et al. Prevalence of complicated grief in a representative population-based sample. J Affect Disord 2011;131:339-43.

3. Prigerson HG, Horowitz MJ, Jacobs SC, et al. Prolonged grief disorder: Psychometric validation of criteria proposed for DSM-V and ICD-11. PLoS Med 2009;6:e1000121.

4. Jordan AH, Litz BT. Prolonged grief disorder: diagnostic, assessment, and treatment considerations. Professional Psychology: Research and Practice. 2014;45:180-7.

5. Prigerson HG, Vanderwerker LC, Maciejewski PK. A case for inclusion of prolonged grief disorder in DSM-V. In: Stroebe MS, Hansson RO, Stroebe W, Schut H. eds. Handbook of bereavement research and practice: advances in theory and intervention. Washington, DC: American Psychological Association Press, 2008:165-86.

6. Kaltman S, Bonanno GA. Trauma and bereavement: examining the impact of sudden and violent deaths. J Anxiety Disord 2003;17:131-47.

7. APA. Diagnostic and statistical manual of mental disorders: DSM-5. 5th edn. Arlington, VA: American Psychiatric Association, 2013.

8. Maercker A, Brewin CR, Bryant RA, et al. Proposals for mental disorders specifically associated with stress in the International Classification of Diseases-11. Lancet 2013;381:1683-5.

9. Doering BK, Eisma MC. Treatment for complicated grief: state of the science and ways forward. Curr Opin Psychiatry 2016;29:286-91.

10. Lichtenthal WG, Nilsson M, Kissane DW, et al. Underutilization of mental health services among bereaved caregivers with prolonged grief disorder. Psychiatr Serv 2011;62:1225-9.

11. Shear K, Frank E, Houck PR, et al. Treatment of complicated grief: a randomized controlled trial. JAMA 2005;293:2601-8.

12. Boelen PA, de Keijser J, van den Hout MA, et al. Treatment of complicated grief: a comparison between cognitive-behavioral therapy and supportive counseling. J Consult Clin Psychol 2007;75:277-84.

13. Eisma MC, Boelen PA, van den Bout J, et al. Internet-based exposure and behavioral activation for complicated grief and rumination: a randomized controlled trial. Behav Ther 2015;46:729-48.

14. Bryant RA, Kenny L, Joscelyne A, et al. Treating prolonged grief disorder: a randomized clinical trial. JAMA Psychiatry 2014;71:1332-9.

15. Maccallum F, Bryant RA. Autobiographical memory following cognitive behaviour therapy for complicated grief. J Behav Ther Exp Psychiatry 2011;42:26-31.

16. Rosner R, Lumbeck G, Geissner E. Effectiveness of an inpatient group therapy for comorbid complicated grief disorder. Psychother Res 2011;21:210-8.

17. Piper WE, Ogrodniczuk JS, Joyce AS, et al. Group composition and group therapy for complicated grief. J Consult Clin Psychol 2007;75:116-25.

18. Supiano KP, Luptak M. Complicated grief in older adults: a randomized controlled trial of complicated grief group therapy. Gerontologist 2014;54:840-56.

19. Stroebe M, Schut $H$. The dual process model of coping with bereavement: rationale and description. Death Stud 1999;23:197-224.

20. Boelen P. Intolerance of uncertainty and emotional distress following the death of a loved one. Anxiety Stress Coping 2010;23:471-8.

21. Ehring T, Watkins ER. Repetitive negative thinking as a transdiagnostic process. Int J Cogn Ther 2008;1:192-205.

22. Boelen PA, Reijntjes A, Smid GE. Concurrent and prospective associations of intolerance of uncertainty with symptoms of prolonged grief, posttraumatic stress, and depression after bereavement. J Anxiety Disord 2016;41:65-72.

23. Eisma MC, Boelen PA, Schut HA, et al. Does worry affect adjustment to bereavement? A longitudinal investigation. Anxiety Stress Coping 2017;30:1-10.

24. Eisma MC, Schut HA, Stroebe MS, et al. Adaptive and maladaptive rumination after loss: a three-wave longitudinal study. Br J Clin Psychol 2015;54:163-80.

25. Eisma MC, Rinck M, Stroebe MS, et al. Rumination and implicit avoidance following bereavement: an approach avoidance task investigation. J Behav Ther Exp Psychiatry 2015;47:84-91.

26. Eisma MC, Stroebe MS, Schut HA, et al. Avoidance processes mediate the relationship between rumination and symptoms of complicated grief and depression following loss. J Abnorm Psychol 2013;122:961-70. 
27. Wells A, Matthews G. Attention and emotion: a clinical perspective. Hove, UK: Lawrence Erlbaum \& Associates, 1994.

28. Bennett $\mathrm{H}$, Wells A. Metacognition, memory disorganization and rumination in posttraumatic stress symptoms. $J$ Anxiety Disord 2010;24:318-25.

29. Wells A. Metacognitive therapy for anxiety and depression. New York: The Guilford Press, 2009.

30. Wenn J, O'Connor M, Breen LJ, et al. Exploratory study of metacognitive beliefs about coping processes in prolonged grief symptomatology. Death Stud 2018:1-11.

31. Wenn J, O'Connor M, Breen LJ, et al. Efficacy of metacognitive therapy for prolonged grief disorder: protocol for a randomised controlled trial. BMJ Open 2015;5:e007221.

32. Eldridge SM, Chan CL, Campbell MJ, et al. CONSORT 2010 statement: extension to randomised pilot and feasibility trials. Pilot Feasibility Stud 2016;2:64.

33. Hudson P, Remedios C, Zordan R, et al. Guidelines for the psychosocial and bereavement support of family caregivers of palliative care patients. J Palliat Med 2012;15:696-702.

34. Tomarken A, Roth A, Holland J, et al. Examining the role of trauma, personality, and meaning in young prolonged grievers. Psychooncology 2012;21:771-7.

35. Sheehan DV, Lecrubier Y, Harnett Sheehan $\mathrm{K}$, et al. The validity of the Mini International Neuropsychiatric Interview (MINI) according to the SCID-P and its reliability. European Psychiatry 1997;12:232-41.

36. Wenn J. Development and testing of metacognitive therapy for prolonged grief disorder: a randomised controlled trial: Curtin University, 2017.

37. Wells A. Detached mindfulness in cognitive therapy: a metacognitive analysis and ten techniques. Journal of Rational-Emotive \& Cognitive-Behavior Therapy 2005;23:337-55.

38. Johnsen I, Dyregrov A, Dyregrov K. Participants with prolonged grief-how do they benefit from grief group participation? Omega 2012:65:87-105.

39. Lecrubier Y, Sheehan DV, Weiller E, et al. The Mini International Neuropsychiatric Interview (MINI). A short diagnostic structured interview: reliability and validity according to the CIDI. European Psychiatry 1997;12:224-31.

40. Sealey M, Breen LJ, O'Connor M, et al. A scoping review of bereavement risk assessment measures: Implications for palliative care. Palliat Med 2015;29:577-89.

41. Lovibond PF, Lovibond SH. The structure of negative emotional states: comparison of the Depression Anxiety Stress Scales (DASS) with the Beck Depression and Anxiety Inventories. Behav Res Ther 1995;33:335-43.

42. Eisma MC, Stroebe MS, Schut HAW, et al. Development and Psychometric Evaluation of the Utrecht Grief Rumination Scale. $J$ Psychopathol Behav Assess 2014;36:165-76.

43. Wells A, Cartwright-Hatton S. A short form of the metacognitions questionnaire: properties of the MCQ-30. Behav Res Ther 2004:42:385-96.

44. Ritsner M, Kurs R, Gibel A, et al. Validity of an abbreviated quality of life enjoyment and satisfaction questionnaire (Q-LES-Q-18) for schizophrenia, schizoaffective, and mood disorder patients. Qual Life Res 2005:14:1693-703.

45. Guy W. ECDEU assessment manual for psychopharmacology. Rockville, MD: US Department of Heath E, and Welfare Public Health Service Alcohol, Drug Abuse, and Mental Health Administration, 1976.

46. Yen M, Lo LH. Examining test-retest reliability: an intra-class correlation approach. Nurs Res 2002;51:59-62.

47. Durlak JA. How to select, calculate, and interpret effect sizes. $J$ Pediatr Psychol 2009;34:917-28.

48. Jacobson NS, Truax P. Clinical significance: a statistical approach to defining meaningful change in psychotherapy research. $J$ Consult Clin Psychol 1991;59:12-19.

49. Breen LJ, O'Connor M. The fundamental paradox in the grief literature: a critical reflection. Omega 2007;55:199-218.

50. Tabachnick BG, Fidell LS. Using multivariate statistics. 5th edn. Boston, USA: Pearson/Allyn \& Bacon, 2007. 